\title{
ON THE STABILITY OF SOLITARY WAVES FOR THE OSTROVSKY EQUATION
}

\author{
BY \\ YUE LIU \\ Department of Mathematics, University of Texas, Arlington, Texas 76019
}

\begin{abstract}
Considered herein is the stability of solitary-wave solutions of the Ostrovsky equation which is an adaptation of the Korteweg-de Vries equation widely used to describe the effect of rotation on the surface and internal solitary waves or the capillary waves. It is shown that the ground state solitary waves are global minimizers of energy functionals with the constrained variational problem and are deduced to be nonlinearly stable for the small effect of rotation. The analysis makes frequent use of the variational properties of the ground states.
\end{abstract}

1. Introduction. The nonlinear dispersive equation

$$
\left(u_{t}-\beta u_{x x x}+\left(u^{2}\right)_{x}\right)_{x}=\gamma u, \quad x \in \mathbf{R},
$$

with $\gamma>0$ originally derived by Ostrovsky [Os] in dimensionless space-time variables $(x, t)$ is a model for the unidirectional propagation of weakly nonlinear long surface and internal waves of small amplitude in a rotating fluid. The liquid is assumed to be incompressible and inviscid. The subscripts in (1.1) denote partial derivatives. Here $x$ is the longitudinal coordinate in the horizontal plane and the free surface $u(t, x)$ has been rendered nondimensional with respect to the constant depth $h$ of the liquid and the gravitational acceleration $g$ and the parameter $\gamma>0$ measures the effect of rotation. The parameter $\beta$ determines the type of dispersion, namely, $\beta<0$ (negative dispersion) for surface and internal waves in the ocean or surface waves in a shallow channel with an uneven bottom and $\beta>0$ (positive dispersion) for capillary waves on the surface of the liquid or for oblique magneto-acoustic waves in plasma [Be], [GaSt], [GiGrSt].

Setting $\gamma=0$ in (1.1) and integrating with respect to $x$ in $\mathbf{R}$ and assuming that the solution $u(t, x)$ and all the derivatives are vanishing at infinity, one obtains the wellknown Korteweg-de Vries equation(KdV)

$$
u_{t}-\beta u_{x x x}+\left(u^{2}\right)_{x}=0, \quad x \in \mathbf{R} .
$$

Received November 20, 2006.

2000 Mathematics Subject Classification. Primary 35Q53, 35B60, 76B25.

Key words and phrases. Ostrovsky equation, solitary waves, stability, weak rotation.

E-mail address: yliu@uta.edu 
Although the structure of (1.1) is very similar to that of (1.2), but unlike KdV equation (1.2), the Ostrovsky equation (1.1) is evidently nonintegrable by the method of inverse scattering transform [GiGrSt], [OsSt]. Invariance, conserved quantities, and solitarywave solutions are fundamental features of (1.1). First, we recall the invariances which can be checked easily by direct computations.

(1) Equation (1.1) is space and time translation invariant. If $u(t, x)$ is a solution of (1.1), then for all $t_{0}>0$ and $x_{0} \in \mathbf{R}, w(t, x)=u\left(t+t_{0}, x+x_{0}\right)$ is also a solution of (1.1).

(2) Equation (1.1) is not Galilean invariant. Moving into a Galilean frame $\xi=x-c t$ with velocity $c$, so that $u(t, x)=w(t, \xi)+c / 2$, this equation transforms to

$$
\left(w_{t}-\beta w_{\xi \xi \xi}+\left(w^{2}\right)_{\xi}\right)_{\xi \xi}=\gamma w_{\xi} .
$$

Second, we recall the corresponding conservation laws:

$$
\begin{gathered}
V(u(t))=\frac{1}{2} \int u^{2}=V(u(0)) \quad(\text { Momentum) }, \\
E(u(t))=\int \frac{\beta}{2} u_{x}^{2}+\frac{\gamma}{2}\left(D_{x}^{-1} u\right)^{2}+\frac{1}{3} u^{3}=E(u(0)) \quad \text { (Energy), } \\
I_{1}(u(t))=\int u=0,
\end{gathered}
$$

and

$$
I_{2}(u(u))=\int x u=0
$$

where the operator $D_{x}^{-k}$ for any natural integer $k$ acts on functions $f \in L_{2}(\mathbf{R})$ such that $\xi^{-k} \hat{f}(\xi) \in L_{2}(\mathbf{R})$. It is defined by the Fourier transform $\left(\widehat{D_{x}^{-k} f}\right)(\xi)=(i \xi)^{-k} \hat{f}(\xi)$.

Another interesting fact is that the structure of (1.1) is also similar to that of the Kadomtsev-Petviashvili equation [KaPe],

$$
\left(u_{t}-\beta u_{x x x}+\left(u^{2}\right)_{x}\right)_{x}=\gamma u_{y y} .
$$

But unlike equation (1.7), (1.1) is one dimensional and equation (1.7) is completely integrable.

In what follows, we denote the norm of $L_{q}(\mathbf{R})$ by $|\cdot|_{q}$ and the Sobolev space $H^{s}(\mathbf{R})$ by $\|\cdot\|_{s}$. We define the space $X_{s}, s \geq 0$, by

$$
X_{s}=\left\{f \in H^{s}(\mathbf{R}), D_{x}^{-1} f \in H^{s}(\mathbf{R})\right\}
$$

equipped with the norm

$$
\|f\|_{X_{s}}=\|f\|_{s}+\left\|D_{x}^{-1} f\right\|_{s} .
$$

Assume $f \in X_{1}$. Note that if $D_{x}^{-1} f \in L_{2}(\mathbf{R})$, then there is a $g \in L_{2}(\mathbf{R})$ such that $f=g_{x}$ at least in the sense of distribution. On the other hand, since $f \in X_{1}$, so $f \in H^{1}(\mathbf{R})$, whence $g_{x x} \in L_{2}(\mathbf{R})$. Thus $g$ lies in $H^{2}(\mathbf{R})$. Actually, a very natural space to look for solutions of the Ostrovsky equation (1.1) is the energy space $X_{1}$ suggested by the conservation law (1.4).

An important ingredient needed in our development is a local existence theory for the initial-value problem. It has been provided by Varlamov and Liu [VaLi]. 
Proposition 1.1 ([VaLi]). Let $u_{0} \in X_{s}, s>3 / 2$, such that $\xi^{-2} \hat{u}_{0} \in L_{2}(\mathbf{R})$. Then there exist $T>0$ and a unique solution $u \in C\left([0, T), X_{s}\right) \cap C^{1}\left([0, T), X_{s-3}\right)$ of $(1.1)$ with the following property: either $T=\infty$ or else $T<\infty$ and $\lim _{t \rightarrow T}\|u(t)\|_{X_{s}}=\infty$. Moreover, we have the conserved functionals (1.3), (1.4), (1.5), and (1.6).

The focus of the development in this section is the solitary-wave solutions of (1.1). Localized, traveling-wave solutions of nonlinear, dispersive wave equations are known in many circumstances to play a central role in the long-time evolution of an initial disturbance.

By a solitary wave, we mean a traveling-wave solution of (1.1) with the form $u(t, x)=$ $\varphi_{c}(x-c t)$ where $c \in \mathbf{R}$ is a given parameter and $\varphi_{c}$, or just denoted by $\varphi$, is a ground state of the stationary problem

$$
\left\{\begin{array}{l}
\left(-\beta \varphi_{x x}-c \varphi+\varphi^{2}\right)_{x}=\gamma D_{x}^{-1} \varphi, \quad x \in \mathbf{R} . \\
\varphi \in X_{1}, \quad \varphi \neq 0,
\end{array}\right.
$$

To define a ground state, we introduce some notation:

$$
\begin{aligned}
L_{c}(u) & =E(u)-c V(u)=\int \frac{\beta}{2} u_{x}^{2}+\frac{\gamma}{2}\left(D_{x}^{-1} u\right)^{2}+\frac{1}{3} u^{3}-\frac{c}{2} \int u^{2}, \\
\Omega_{c} & =\left\{u \in X_{1}, \quad u \neq 0, \quad L_{c}^{\prime}(u)=0\right\}=\text { the set of the solutions for (1.8), }
\end{aligned}
$$

and

$$
G_{c}=\left\{u \in \Omega_{c}, \quad L_{c}(u) \leq L_{c}(v), \forall v \in \Omega_{c}\right\}
$$

where $G_{c}$ is called the set of the ground states of (1.8).

It was proved by Liu and Varlamov [LiVa] that if $\beta>0$ and $c<2 \sqrt{\gamma \beta}$, then $G_{c}$ is not empty.

It is known that equation (1.2) has a unique soliton up to translation in the form

$$
\varphi_{c}(x)=\frac{3 c}{2} \operatorname{sech}^{2}\left(\frac{1}{2} \sqrt{\frac{c}{|\beta|}} x\right)
$$

for any $\beta<0$ and $c>0$. However, it is shown in [GaSt], [GiGrSt], [LiVa] that equation (1.8) does not admit any nontrivial solitary waves in the energy space provided $\beta<0$ and for some positive $c$ with $c<\sqrt{140 \gamma|\beta|}$. Hence, the question of how an initial perturbation in the form of a $\mathrm{KdV}$ soliton will be destroyed is more interesting to investigate. On the other hand, it is known that the KdV equation (1.2) does not have any nontrivial solitary waves in the energy space, when $\beta>0$ and $c>0$. However, unlike (1.2), the equation (1.1) does have solitary-wave solutions even for some positive $c$ satisfying $c<2 \sqrt{\gamma \beta}$ with any $\beta>0$ [LiVa]. This notable property of the equation makes the search of its stability of solitary waves highly desirable.

Define the number $I_{q}$ by

$$
I_{q}=\inf \left\{E(u): \quad u \in X_{1}, V(u)=q\right\},
$$

where $q=V\left(\varphi_{c}\right)$ for some $\varphi_{c} \in G_{c}$. The set of minimizers for $I_{q}$ is

$$
\Sigma_{q}=\left\{g \in X_{1}: \quad E(g)=I_{q}, V(g)=q\right\} .
$$


The Euler-Lagrange equation for the constrained minimization problem solved by the functions in $\Sigma_{q}$ is

$$
\delta E(g)=\lambda \delta V(g),
$$

where $\lambda$ is the Lagrange multiplier. It is found that if $g \in \Sigma_{q}$, then $g$ is a solution of (1.8) with wave speed $c=\lambda$.

In this paper, we are considering nonlinear stability with respect to arbitrary perturbations of set $\Sigma_{q}$, since solitary waves might not be unique up to translation.

Definition 1.2. A set $S \subset X$ is called $X$-stable with respect to equation (1.1) if for a given $\epsilon>0$, there exists such a $\delta>0$ such that for any $u_{0} \in X \cap X_{s}, s>3 / 2$, with

$$
\inf _{v \in S}\left\|u_{0}-v\right\|_{X}<\delta
$$

the solution $u(t)$ of (1.1) with initial value $u_{0}$ can be extended to a solution in $C([0, \infty)$, $\left.X \cap X_{s}\right)$ and satisfies

$$
\inf _{v \in S}\|u(t)-v\|_{X}<\epsilon
$$

for all $t \geq 0$. Otherwise we say that the set $S$ is $X$-unstable. The principal result of the present paper is the following.

Theorem 1.3 (Nonlinear stability). Let $\beta>0$ and $c<2 \sqrt{\gamma \beta}$. Then there exists $\gamma_{0}>0$ such that for any $\gamma<\gamma_{0}$, the set $\Sigma_{q}$ is $X_{1}$-stable.

Stability of the set of ground states $G_{c}$ was proved in [LiVa] under the assumption of convexity of the action $d(c)=E\left(\varphi_{c}\right)-c V\left(\varphi_{c}\right)$ with $\varphi_{c} \in G_{c}$. It can be done by showing the solitary wave $\varphi_{c}$ is a local constrained minimizer of a Hamiltonian functional with this condition of $d(c)$. However, being different from the case of the KdV or KP equations, the scaling and dilation technique does not give the description of action $d(c)$ explicitly. To remove this assumption of convexity of $d(c)$, an alternate approach to proving stability of solitary waves is that, rather than using local analysis, we start instead with the constrained variational problem for global minimizer. The proof of Theorem 1.3 is to employ a modification of the concentration compactness principle [Lio] together with a rigorous justification of global analysis of minimizers, but the small effect of rotation $\gamma>0$ is required. An easy corollary of proving such a minimizer is that the set of global minimizers is a stable set for the associated initial value problem.

The remainder of the paper is organized as follows. In Section 2, we study the properties of the ground states of (1.8). We consider the associated minimization problem and employ a refined Fatou Lemma [BrLi] to obtain again the set of ground states. In Section 3, we show in detail based on an outline of [Lio] how it is used to prove stability of ground states of (1.1). Finally, in Section 4, as a consequence of global minimizers of functional $E$, we are able to prove dynamical stability of global minimizers (Theorem $1.3)$.

2. The solitary waves. The existence and the qualitative properties of solitary waves for equation (1.1) are known in part. For example, in [LiVa], Liu and Varlamov investigate the existence, regularity and decay estimates of solitary waves of (1.8) .

We start with the existence and nonexistence of solutions of solitary waves of (1.1). 
Proposition 2.1. Suppose $\beta<0$ and $c<\sqrt{140 \gamma|\beta|}$. Then equation (1.1) does not admit any nontrivial solitary-wave solutions $\varphi_{c} \in X_{1}$.

Proof. The proof is given in [LiVa, Theorem 2.1] by using the Pohojaev-type identities.

REMARK. It is also possible to show the nonexistence of any nontrivial solitary-waves in $X_{1}$, if $\beta>0$ and $c \geq 2 \sqrt{\gamma \beta}$. In fact, suppose $u(t, x)=\varphi_{c}(x-c t)$ is a nontrivial solitarywave solution of (1.1) satisfying equation (1.8). Since $\varphi_{c} \in H^{1}$, a bootstrap argument shows the solution $\varphi_{c} \in H^{\infty}$ and $\varphi_{c} \rightarrow 0$ together with its derivatives as $|x| \rightarrow \infty$. Thus, to consider the asymptotic state of the solution $\varphi_{c}$ of (1.8), it thus suffices to study the solution $\phi_{c}$ of the linearized equation by neglecting the nonlinear term $\left(\varphi_{c}^{2}\right)_{x x}$, that is,

$$
\beta \partial_{x}^{4} \phi_{c}-c \partial_{x}^{2} \phi_{c}-\gamma \phi_{c}=0
$$

The characteristic equation of (2.1) is

$$
\beta \lambda^{4}+\lambda^{2}+\gamma=0
$$

and the roots of (2.2) have the form

$$
\lambda^{2}=\frac{-c \pm \sqrt{c^{2}-4 \gamma \beta}}{2 \beta} .
$$

In the case of $\beta>0$ and $c \geq 2 \sqrt{\gamma \beta}$, we obtain from (2.3) that there are only pure imaginary roots of (2.2). It turns out that the solution $\phi_{c}$ of (2.1) does not vanish as $|x| \rightarrow \infty$. Since $\varphi_{c} \sim \phi_{c}$, as $|x| \rightarrow \infty$, this contradicts the fact that $\varphi_{c}$ vanishes at infinity. Note for $\beta<0$ that this result of the nonexistence of solitary waves is sharp in the following sense.

Proposition 2.2. Assume that $\beta>0$ and $c<2 \sqrt{\gamma \beta}$. Then there exists a ground state $\varphi_{c}$ of (1.8), that is, the solitary-wave solution of (1.1) in $X_{1}$.

Proof. See Theorem 2.3 in [LiVa].

REMARK. It is shown in [LiVa] that $x^{2}\left|\varphi_{c}\right|_{\infty} \leq C_{0}$. In fact, we have an optimal decay result by showing that the solution $\varphi_{c}$ of (1.8) decays to zero exponentially as $|x| \rightarrow \infty$. To see this, first we find that the solution $\varphi_{c}$ of (1.8) is in $H^{\infty}$ and therefore $\varphi_{c} \in C^{5}(\mathbf{R})$ is a classical solution of (1.8). Next, we rewrite (1.8) as a system for $\vec{\varphi}_{c}=\left\langle\varphi_{c}, \varphi_{2}, \varphi_{3}, \varphi_{4}\right\rangle$, i.e.

where

$$
\frac{d \vec{\varphi}_{c}}{d x}=\mathbf{A} \vec{\varphi}_{c}+\mathbf{N}\left(\vec{\varphi}_{c}\right)
$$

$$
\mathbf{A}=\left(\begin{array}{cccc}
0 & 1 & 0 & 0 \\
0 & 0 & 1 & 0 \\
0 & 0 & 0 & 1 \\
-\frac{\gamma}{\beta} & 0 & -\frac{c}{\beta} & 0
\end{array}\right)
$$

and $\mathbf{N}\left(\varphi_{c}\right)=\left\langle 0,0,0, \frac{2}{\beta}\left(\varphi_{2}^{2}+\varphi_{c} \varphi_{3}\right)\right\rangle$. From the characteristic equation (2.2) of $\mathbf{A}$, it is easy to verify that $\mathbf{A}$ has two eigenvalues with positive real parts and two with negative real parts provided $\beta>0$ and $c<2 \sqrt{\gamma \beta}$. Hence the exponential decay of $\varphi_{c}$ follows 
from the stable-manifold theorem if one can show that $\varphi_{c}$ and its first three derivatives approach zero as $x \rightarrow+\infty$. Since $\varphi_{c} \in H^{4}(\mathbf{R})$, it is easy to show by the fact

$$
\left\|\varphi_{c}\right\|_{C^{3}([n, n+1])} \leq C_{0}\left\|\varphi_{c}\right\|_{H^{4}([n, n+1])} \rightarrow 0, \quad \text { as } \quad n \rightarrow \infty .
$$

We next define

$$
P(u)=\int \beta u_{x}^{2}+\gamma\left(D_{x}^{-1} u\right)^{2}-c u^{2}+u^{3} .
$$

Then we can rewrite (1.9) as

$$
L_{c}(u)=\frac{1}{2} P(u)-\frac{1}{6} \int u^{3} .
$$

The following result gives a description of ground state as a minimizer of $L_{c}$ with constraint $P(u)=0$. This property of ground states will be used to prove the stability result in Theorem 1.3.

Proposition 2.3. Assume $\beta>0$ and $c<2 \sqrt{\gamma \beta}$. Then there exists $\varphi_{c} \in X_{1}$ satisfying $P\left(\varphi_{c}\right)=0$ such that

$$
L_{c}\left(\varphi_{c}\right)=\inf \left\{L_{c}(u), u \in X_{1}, u \neq 0, P(u)=0\right\} .
$$

Such a minimizer $\varphi_{c}$ of (2.6) is a ground state of (1.8), that is, $\varphi_{c} \in G_{c}$. Moreover, if any $\phi \in G_{c}$, then $\phi$ is a solution of the minimization problem (2.6).

The proof of Proposition 2.3 is approached via a series of lemmas.

Lemma 2.4 (Fröhlich, Lieb and Loss [FrLiLo]). Let $1<\alpha<\mu<\nu$ and let $f(x)$ be a measurable function on $\mathbf{R}$ such that $|f|_{\alpha} \leq C_{\alpha},|f|_{\mu} \geq C_{\mu}>0$ and $|f|_{\nu} \leq C_{\nu}$ for some positive constants $C_{\alpha}, C_{\mu}$ and $C_{\nu}$. Then for some positive constants $\eta$ and $c_{0}$ the Lebesgue measure meas $\{x \in \mathbf{R},|f(x)|>\eta\} \geq C_{0}$, where $c_{0}$ depends on $\alpha, \mu, \nu, C_{\alpha}, C_{\mu}$, and $C_{\nu}$, but not on $f$.

Lemma 2.5 (Lieb [Lie]). Let $\left\{f_{j}\right\}$ be a bounded sequence in $H^{1}(\mathbf{R})$ such that

$$
\operatorname{meas}\left\{x ;\left|f_{j}(x)\right|>\eta\right\} \geq C_{0}
$$

for some positive constants $\eta$ and $C_{0}>0$. Then there exists a sequence $\left\{y_{j}\right\} \in \mathbf{R}$ such that for some subsequence (still denoted by the same letter) and $f \in H^{1}, f_{j}\left(\cdot-y_{j}\right) \rightarrow f \not \equiv 0$ weakly in $H^{1}(\mathbf{R})$.

The following lemma is called the refined Fatou lemma due to Brézis and Lieb [BrLi].

Lemma 2.6. Let $\left\{f_{j}\right\}$ be a bounded sequence in $L_{r}(\mathbf{R})$ for $0<r<\infty$. If $f_{j} \rightarrow f$ a.e. in $\mathbf{R}$, then

$$
\left|f_{j}\right|_{r}^{r}-\left|f_{j}-f\right|_{r}^{r}-|f|_{r}^{r} \rightarrow 0
$$

as $j \rightarrow \infty$. When $r=2$, the assumption that $f_{j} \rightarrow f$ a.e. in $\mathbf{R}$ is not necessary.

Now we are in the position to prove Proposition 2.3 .

Proof of Proposition 2.3. We define

$$
d_{c}=\inf \left\{L_{c}(u), P(u)=0\right\}
$$


and

$$
m_{c}=\inf \left\{L_{c}^{1}(u), P(u) \leq 0\right\}
$$

where

$$
L_{c}^{1}(u)=L_{c}(u)-\frac{1}{3} P(u)=\frac{1}{6}\left(\beta \int u_{x}^{2}+\gamma \int\left(D_{x}^{-1} u\right)^{2}-c \int u^{2}\right) .
$$

By the assumption of Proposition 2.3 and the fact that $\int u^{2}=-\int u_{x} D_{x}^{-1} u$, it is easy to verify that

$$
L_{c}^{1}(u) \geq C_{0}(\beta, \gamma, c)\left(\left|D_{x}^{-1} u\right|_{2}^{2}+\left|u_{x}\right|_{2}^{2}\right), \quad \forall u \in X_{1}
$$

with some constant $C_{0}(\beta, \gamma, c)$. We first claim that $d_{c}=m_{c}$. Suppose $u \in X_{1}$ satisfies that $P(u)<0$. Since

$$
P(\lambda u)=\lambda^{2}\left(\beta \int u_{x}^{2}+\gamma \int\left(D_{x}^{-1} u\right)^{2}-c \int u^{2}\right)+\lambda^{3} \int u^{3}>0
$$

for some sufficiently small $\lambda>0$, there exists $\lambda_{0} \in(0,1)$ such that $P\left(\lambda_{0} u\right)=0$. Hence it follows from the definition of $m_{c}$ that

$$
\begin{aligned}
d_{c} & \leq L_{c}\left(\lambda_{0} u\right)=\lambda_{0}^{2}\left(\frac{\beta}{2} \int u_{x}^{2}+\frac{\gamma}{2} \int\left(D_{x}^{-1} u\right)^{2}-\frac{c}{2} \int u^{2}\right)+\frac{\lambda_{0}^{3}}{3} \int u^{3} \\
& =\lambda_{0}^{2}\left(\frac{\beta}{2} \int u_{x}^{2}+\frac{\gamma}{2} \int\left(D_{x}^{-1} u\right)^{2}-\frac{c}{2} \int u^{2}\right)-\frac{\lambda_{0}^{3}}{3}\left(\beta \int u_{x}^{2}+\gamma \int\left(D_{x}^{-1} u\right)^{2}-c \int u^{2}\right) \\
& =\frac{1}{6} \lambda_{0}^{2}\left(\beta \int u_{x}^{2}+\gamma \int\left(D_{x}^{-1} u\right)^{2}-c \int u^{2}\right) \\
& <\frac{1}{6}\left(\beta \int u_{x}^{2}+\gamma \int\left(D_{x}^{-1} u\right)^{2}-c \int u^{2}\right) \\
& =L_{c}^{1}(u) .
\end{aligned}
$$

This implies that $d_{c} \leq m_{c}$ and therefore $d_{c}=m_{c}$. As a consequence, to show the existence of a minimizer of $d_{c}$, it suffices to show there exists a minimizer of $m_{c}$. Let $\left\{u_{n}\right\}$ be a minimizing sequence satisfying $\lim _{n \rightarrow \infty} L_{c}^{1}\left(u_{n}\right)=m_{c}$ with $P\left(u_{n}\right) \leq 0$. Then by the estimate (2.10), the minimizing sequence $\left\{u_{n}\right\}$ is bounded in $X_{1}$. Hence, there exists a function $u_{0} \in X_{1}$ such that a subsequence (still denoted by $u_{n}$ ) weakly converges to $u_{0}$ in $X_{1}$. Next we show that such a solution $u_{0}$ is a minimizer of $m_{c}$, that is, $L_{c}^{1}\left(u_{0}\right)=m_{c}$ with $P\left(u_{0}\right)$. Toward this end, we split the proof into five steps.

Step 1. $\inf _{n}\left|u_{n}\right|_{3}^{3}>0$.

Proof. To prove this statement, we argue by contradiction. If $\inf _{n}\left|u_{n}\right|_{3}^{3}=0$, then there exists a subsequence, still denoted by $u_{n}$, such that $u_{n} \neq 0, \forall n \geq 1$ and $\lim _{n \rightarrow \infty}\left|u_{n}\right|_{3}^{3}=0$. It follows from $P\left(u_{n}\right) \leq 0$ that

$$
\beta \int\left(\partial_{x} u_{n}\right)^{2}+\gamma \int\left(D_{x}^{-1} u_{n}\right)^{2}-c \int u_{n}^{2} \leq-\int u_{n}^{3} \leq\left|u_{n}\right|_{3}^{3} \rightarrow 0
$$


as $n \rightarrow \infty$. On the other hand, using the Sobolev embedding, we have

$$
\begin{aligned}
\left|u_{n}\right|_{3}^{3} & \leq 4 \sqrt{2}\left|u_{n}\right|_{2}^{\frac{3}{2}}\left|D_{x}^{-1} u_{n}\right|_{2}^{\frac{1}{2}}\left|\partial_{x} u_{n}\right|_{2} \\
& \leq C_{0}\left(\left|D_{x}^{-1} u_{n}\right|_{2}^{2}+\left|\partial_{x} u_{n}\right|_{2}^{2}\right)^{\frac{3}{4}}\left|D_{x}^{-1} u_{n}\right|_{2}^{\frac{1}{2}}\left|\partial_{x} u_{n}\right|_{2} \\
& \leq C_{0}\left(\left|D_{x}^{-1} u_{n}\right|_{2}^{2}+\left|\partial_{x} u_{n}\right|_{2}^{2}\right)^{\frac{3}{2}} \\
& \leq C_{0}\left(\beta\left|\partial_{x} u_{n}\right|_{2}^{2}+\gamma\left|D_{x}^{-1} u_{n}\right|_{2}^{2}-C\left|u_{n}\right|_{2}^{2}\right)^{\frac{3}{2}}
\end{aligned}
$$

where $C_{0}$ represents various constants depending only on $\beta, \gamma$, and $c$. Combining (2.13) with (2.15) yields

$$
\left(\beta\left|\partial_{x} u_{n}\right|_{2}^{2}+\gamma\left|D_{x}^{-1} u_{n}\right|_{2}^{2}-c\left|u_{n}\right|_{2}^{2}\right)\left(1-C_{0}\left(\beta\left|\partial_{x} u_{n}\right|_{2}^{2}+\gamma\left|D_{x}^{-1} u_{n}\right|_{2}^{2}-c\left|u_{n}\right|_{2}^{2}\right)^{\frac{1}{2}}\right) \leq 0 .
$$

This implies that

$$
C_{0}\left(\beta\left|\partial_{x} u_{n}\right|_{2}^{2}+\gamma\left|D_{x}^{-1} u_{n}\right|_{2}^{2}-c\left|u_{n}\right|_{2}^{2}\right) \geq 1
$$

which contradicts $(2.13)$.

Step 2. The solution $u_{0} \neq 0$, a.e. in $\mathbf{R}$.

Proof. Using Step 1, it is simply an application of Lemma 2.4 and Lemma 2.5 for the choice of $\alpha=2, \mu=3$, and $\nu=4$.

Step 3. $L_{c}^{1}\left(u_{0}\right)=m_{c}$.

Proof. By Lemma 2.6, we deduce that

$$
L_{c}^{1}\left(u_{n}\right)-L_{c}^{1}\left(u_{n}-u_{0}\right)-L_{c}^{1}\left(u_{0}\right) \longrightarrow 0,
$$

as $n \rightarrow \infty$. On the other hand, we have

$$
\begin{aligned}
& \int u_{n}^{3}-\int\left(u_{n}-u_{0}\right)^{3}-\int u_{0}^{3}=-\int\left(-3 u_{n}^{2} u_{0}+3 u_{n} u_{0}^{2}-u_{0}^{3}\right) \\
& =3 \int u_{n}^{2} u_{0}-3 \int u_{n} u_{0}^{2} \longrightarrow 3 \int u_{0}^{3}-3 \int u_{0} u_{0}^{2}=0,
\end{aligned}
$$

as $n \rightarrow \infty$, since $u_{n} \rightarrow u_{0}$ weakly in $X_{1}$ and $u_{n} \rightarrow u_{0}$ a.e. in $\mathbf{R}$ imply that $u_{n} \rightarrow u_{0}$ weakly in $L_{4}$ and $u_{n}^{2} \rightarrow u_{0}^{2}$ weakly in $L_{2}$.

It follows from (2.19) that

$$
P\left(u_{n}\right)-P\left(u_{n}-u_{0}\right)-P\left(u_{0}\right) \longrightarrow 0
$$

as $n \rightarrow \infty$. Now we claim that $P\left(u_{0}\right) \leq 0$. Toward this end, we argue by contradiction. Suppose $P\left(u_{0}\right)>0$. Then from the fact that $P\left(u_{n}\right) \leq 0, \forall n$ and (2.19), we obtain that $P\left(u_{n}-u_{0}\right) \leq 0$, as $n \rightarrow \infty$. By the definition of $m_{c}$, it turns out that $L_{c}^{1}\left(u_{n}-u_{0}\right) \geq m_{c}$. But $L_{c}^{1}\left(u_{n}\right) \rightarrow m_{c}$ as $n \rightarrow \infty$. Hence it can be deduced from $(2.17)$ that $L_{c}^{1}\left(u_{0}\right) \leq 0$, that is

Since

$$
\beta \int\left(\partial_{x} u_{0}\right)^{2}+\gamma \int\left(D_{x}^{-1} u_{0}\right)^{2}-c \int u_{0}^{2} \leq 0
$$

$$
C(\beta, \gamma, c)\left(\int\left(\partial_{x} u_{0}\right)^{2}+\int\left(D_{x}^{-1} u_{0}\right)^{2}\right) \leq \beta \int\left(\partial_{x} u_{0}\right)^{2}+\gamma \int\left(D_{x}^{-1} u_{0}\right)^{2}-c \int u_{0}^{2},
$$

it implies from (2.21) that $u_{0}=0$ a.e. in $\mathbf{R}$, which is a contradiction. Hence $P\left(u_{0}\right) \leq$ 0 . 
Step 4. $P\left(u_{0}\right)=0$.

Proof. Again we argue by contradiction. Suppose $P\left(u_{0}\right)<0$. Then choosing some sufficiently small $\lambda>0$, we have

$$
P\left(\lambda u_{0}\right)=\lambda^{2}\left(\beta \int\left(\partial_{x} u_{0}\right)^{2}+\gamma \int\left(D_{x}^{-1} u_{0}\right)^{2}-c \int u_{0}^{2}\right)+\lambda^{3} \int u_{0}^{3}>0 .
$$

By continuity of $P$, there exists $\lambda_{0} \in(0,1)$ such that

$$
P\left(\lambda_{0} u_{0}\right)=0 .
$$

Applying (2.16) to the minimization problem of $m_{c}$ would yield a contradiction, that is,

$$
\begin{aligned}
m_{c} & \leq L_{c}^{1}\left(\lambda_{0} u_{0}\right)=\frac{1}{6} \lambda_{0}^{2}\left(\beta \int\left(\partial_{x} u_{0}\right)^{2}+\gamma \int\left(D_{x}^{-1} u_{0}\right)^{2}-c \int u_{0}^{2}\right) \\
& <\frac{1}{6}\left(\beta \int\left(\partial_{x} u_{0}\right)^{2}+\gamma \int\left(D_{x}^{-1} u_{0}\right)^{2}-c \int u_{0}^{2}\right)=m_{c},
\end{aligned}
$$

and hence, $P\left(u_{0}\right)=0$.

Step 5. $u_{0} \in G_{c}$, that is, $u_{0}$ is a ground state of (1.8).

Proof. It follows from the results in Step 3 and Step 4 that

$$
m_{c}=d_{c}=\inf \left\{L_{c}(u), P(u)=0\right\}=L_{c}\left(u_{0}\right) .
$$

Hence, by the Lagrange multiplier principle, there exists $\mu \in \mathbf{R}$ such that

$$
L_{c}^{\prime}\left(u_{0}\right)+\mu P^{\prime}\left(u_{0}\right)=0
$$

where $L_{c}^{\prime}\left(u_{0}\right)$ and $P^{\prime}\left(u_{0}\right)$ are the Fréchet derivatives of $L_{c}$ and $P$ at $u_{0}$. It is thereby inferred from (2.26) that

$$
\left\langle L_{c}^{\prime}\left(u_{0}\right), u_{0}\right\rangle=-\mu\left\langle P^{\prime}\left(u_{0}\right), u_{0}\right\rangle
$$

But

$$
\left\langle L_{c}^{\prime}\left(u_{0}\right), u_{0}\right\rangle=P\left(u_{0}\right)=0
$$

and

$$
\begin{aligned}
\left\langle P^{\prime}\left(u_{0}\right), u_{0}\right\rangle= & 2\left(\beta \int\left(\partial_{x} u_{0}\right)^{2}+\gamma \int\left(D_{x}^{-1} u_{0}\right)^{2}-c \int u_{0}^{2}\right)+3 \int u_{0}^{3} \\
= & 2\left(\beta \int\left(\partial_{x} u_{0}\right)^{2}+\gamma \int\left(D_{x}^{-1} u_{0}\right)^{2}-c \int u_{0}^{2}\right) \\
& -3\left(\beta \int\left(\partial_{x} u_{0}\right)^{2}+\gamma \int\left(D_{x}^{-1} u_{0}\right)^{2}-c \int u_{0}^{2}\right) \\
= & -\left(\beta \int\left(\partial_{x} u_{0}\right)^{2}+\gamma \int\left(D_{x}^{-1} u_{0}\right)^{2}-c \int u_{0}^{2}\right)<0 .
\end{aligned}
$$

It then follows from (2.26) and (2.27) that $\lambda=0$ and $L_{c}^{\prime}\left(u_{0}\right)=0$. On the other hand, for any nonzero $v \in X_{1}$, if $L_{c}^{\prime}(v)=0$, then we have $P(v)=\left.\frac{d}{d \lambda} L_{c}(\lambda v)\right|_{\lambda=1}=\left\langle L_{c}^{\prime}(v), v\right\rangle=$ 0 . By the definition of $d_{c}$, we thus deduce that $L_{c}\left(u_{0}\right) \leq L_{c}(v)$, namely, $u_{0} \in G_{c}$. Furthermore, if $\varphi_{c} \in G_{c}$, then $L_{c}^{\prime}\left(\varphi_{c}\right)=0$ and $L_{c}\left(\varphi_{c}\right) \leq L_{c}(v)$ for any $v \in X_{1}$ satisfying $L_{c}^{\prime}(u)=0$. Since

$$
P(v)=\left.\frac{d}{d \lambda} L_{c}(\lambda v)\right|_{\lambda=1}=\left\langle L_{c}^{\prime}(v), v\right\rangle,
$$


for any $v \in X_{1}$, it follows that $\varphi_{c}$ is a minimizer of $d_{c}$, and $d_{c}=m_{c}=L_{c}\left(\varphi_{c}\right)=d(c)$. This completes the proof of Proposition 2.3.

3. Minimizers of the energy. It was proved in $[\mathrm{LiVa}]$ that the set of the ground states $G_{c}$ is nonlinearly stable in the energy space $X_{1}$ under the assumption of $d^{\prime \prime}(c)>0$, since the scaling and dilation technique does not give the description of action $d(c)$ explicitly in terms of the wave's speed $c$. We will now use another characterization of the ground states solutions of (1.8) in order to show that the set of global minimizers is nonlinearly stable. The proof of the existence of a ground state as well as its implications for stability follows the outline by Cazenave and Lions [CaLi] concerning the stability of standing waves for the nonlinear Schrödinger equation. In this regard, we consider the ground states solutions characterized as minimizers of the energy function $E$ constrained by the constant momentum $V$. The argument is based on an outline of the concentration compactness lemma [Lio]. But the difficult part of applying the concentration compactness lemma is that the scaling and dilation technique cannot give the exact description of the minimization of the energy $E$ with the constant constraint of the momentum $V$. To avoid this difficulty, we have to restrict the effect of rotation $\gamma$ to be small enough.

Suppose that $\varphi_{c} \in G_{c}$ for $c<2 \sqrt{\gamma \beta}$ and $\beta>0$. Define $q=V\left(\varphi_{c}\right)$. The central role will be played by minimization problem $I_{q}$ defined by

$$
I_{q}=\inf \left\{E(u): u \in X_{1}, V(u)=q\right\} .
$$

The set of minimizers for $I_{q}$ is defined by

$$
\Sigma_{q}=\left\{u \in X_{1}: E(u)=I_{q}, \quad V(u)=q\right\},
$$

and the minimizing sequence for $I_{q}$ is any sequence $\left\{u_{n}\right\}$ of functions in $X_{1}$ satisfying

$$
V\left(u_{n}\right)=q, \quad \forall n \geq 1
$$

and

$$
\lim _{n \rightarrow \infty} E\left(u_{n}\right)=I_{q}
$$

The stability of the set $\Sigma_{q}$ is a natural consequence of the following theorem.

TheOREm 3.1. Let $\beta>0$ and $c<2 \sqrt{\gamma \beta}$. Then

(1) there exists $\gamma_{0}>0$ such that if $0<\gamma<\gamma_{0}$, then the set $\Sigma_{q}$ is not empty,

(2) any minimizing sequence $\left\{u_{n}\right\}$ for $I_{q}$ is relatively compact in $X_{1}$ up to translations, that is, there exists a sequence $\left\{y_{n}\right\}$ and an element $g \in \Sigma_{q}$ such that $u_{n}\left(\cdot+y_{n}\right)$ has a subsequence converging strongly in $X_{1}$ to $g$,

(3) $\lim _{n \rightarrow \infty} \inf _{g \in \Sigma_{q}, y \in \mathbf{R}}\left\|u_{n}(\cdot+y)-g\right\|_{X_{1}}=0$,

(4) $\lim _{n \rightarrow \infty} \inf _{g \in \Sigma_{q}}\left\|u_{n}-g\right\|_{X_{1}}=0$, and

(5) each $g \in \Sigma_{q}$ with $P(g)=0$ is a ground-state solution of (1.1), where $P$ is defined in (2.4).

Let us denote by $I_{\mu}$, for $\mu>0$, the minimization problem

$$
I_{\mu}=\inf \left\{E(u): u \in X_{1}, V(u)=\mu\right\} .
$$


Recall that

and

$$
E(u)=\frac{\beta}{2} \int u_{x}^{2}+\frac{\gamma}{2} \int\left(D_{x}^{-1} u\right)^{2}+\frac{1}{3} \int u^{3}
$$

$$
V(u)=\frac{1}{2} \int u^{2}
$$

The proof of the theorem is approached via a series of lemmas.

Lemma 3.2. For all $\mu>0$, there exists $\gamma_{0}>0$, such that if $0<\gamma<\gamma_{0}$, then $-\infty<I_{\mu}<$ 0 .

Proof. Let $\phi$ be a ground state in $G_{c_{1}}$ with $\gamma=1$ for $c_{1}<2 \sqrt{\beta}$. Then

$$
-\int \phi^{3}=\beta \int\left(\partial_{x} \phi\right)^{2}+\int\left(D_{x}^{-1} \phi\right)^{2}-c_{1} \int \phi^{2} \geq\left(2 \sqrt{\beta}-c_{1}\right) \int \phi^{2}>0 .
$$

For $\mu \leq 1$ we define the function $w=a \phi$, where $a>0$ is chosen so that $V(w)=\mu$ and $\int w^{3}=a^{3} \int \phi^{3}<0$. For each $\eta>0$, define the function $w_{\eta}$ by

$$
w_{\eta}(x)=\sqrt{\eta} w(\eta x) .
$$

Then for all $\eta>0$, we have $V\left(w_{\eta}\right)=V(w)=\mu$ and

$$
E\left(w_{\eta}\right)=\frac{\eta^{2} \beta}{2} \int\left(\partial_{x} w\right)^{2}+\frac{\eta^{-2} \gamma}{2} \int\left(D_{x}^{-1} w\right)^{2}+\frac{\eta^{\frac{1}{2}}}{3} \int w^{3} .
$$

If we choose $\eta=\gamma^{\frac{1}{4}}>0$ such that $\gamma \eta^{-2}=\eta^{2}$, then by taking $\gamma_{0}>0$ sufficiently small, we get for all $\gamma<\gamma_{0}$,

$$
E\left(w_{\eta}\right)=\eta^{\frac{1}{2}}\left(\frac{\eta^{\frac{3}{2}} \beta}{2} \int\left(\partial_{x} w\right)^{2}+\frac{\eta^{\frac{3}{2}}}{2} \int\left(D_{x}^{-1} w\right)^{2}+\frac{1}{3} \int w^{3}\right)<0,
$$

and it follows that $I_{\mu}<0$ for any $\mu \leq 1$.

It remains to show that $I_{\mu}<0$ for any $\mu>1$ as well. To see this, let $w_{1}$ be the function constructed as above for $\mu=1$ so that $E\left(w_{1}\right)<0$ and $V\left(w_{1}\right)=1$. For any given $\mu>1$, we define $w_{2}=\sqrt{\mu} w_{1}$. Since $\sqrt{\mu}>1$ and $\int w_{1}^{3}<0$, we have

$$
\begin{aligned}
E\left(w_{2}\right) & =\frac{\mu \beta}{2} \int\left(\partial_{x} w_{1}\right)^{2}+\frac{\mu \gamma}{2} \int\left(D_{x}^{-1} w_{1}\right)^{2}+\frac{\mu^{\frac{3}{2}}}{3} \int w_{1}^{3} \\
& \leq \mu\left(\frac{\beta}{2} \int\left(\partial_{x} w_{1}\right)^{2}+\frac{\gamma}{2} \int\left(D_{x}^{-1} w_{1}\right)^{2}+\frac{1}{3} \int w_{1}^{3}\right)=\mu E\left(w_{1}\right)<0 .
\end{aligned}
$$

But $V\left(w_{2}\right)=\mu V\left(w_{1}\right)=\mu$, and so it has been proved that $I_{\mu}<0$ for all $\mu>0$.

To prove that $I_{\mu}>-\infty$, let $v$ denote any function in $X_{1}$ satisfying $V(v)=\mu$. Note from a standard Sobolev embedding and the interpolation theorem that we have

$$
\left|\int v^{3}\right| \leq|v|_{3}^{3} \leq C_{0}\|v\|_{\frac{1}{6}}^{3} \leq C_{0}|v|_{2}^{\frac{5}{2}}\|v\|_{1}^{\frac{1}{2}}
$$

where $C_{0}$ denotes a universal constant which is independent of $v$. Then applying Young's inequality

$$
\left|\int v^{3}\right| \leq \epsilon\|v\|_{1}^{2}+C_{\epsilon}|v|_{2}^{\frac{10}{3}}
$$


for a small $\epsilon>0$ yields

$$
\begin{aligned}
E(v) & =E(v)+\beta V(v)-\beta V(v) \\
& =\frac{\beta}{2} \int v_{x}^{2}+\frac{\gamma}{2} \int\left(D_{x}^{-1} v\right)^{2}+\frac{\beta}{2} \int v^{2}-\frac{1}{3} \int v^{3}-\beta \mu \\
& \geq \frac{\min \{\beta, \gamma\}}{2}\|v\|_{X}^{2}-\frac{\min \{\beta, \gamma\}}{4}\|v\|_{1}^{2}-C_{\beta, \gamma}|v|_{2}^{\frac{10}{3}}-\beta \mu \\
& \geq \frac{\min \{\beta, \gamma\}}{4}\|v\|_{X}^{2}-2 C_{\beta, \gamma} \mu^{\frac{5}{2}}-\beta \mu>-\infty
\end{aligned}
$$

where $\epsilon$ is chosen sufficiently small such that $0<\epsilon<\frac{\min \{\beta, \gamma\}}{4}$.

Lemma 3.3. Let $\beta>0$ and $c<2 \sqrt{\gamma \beta}$. If $\left\{u_{n}\right\}$ is a minimizing sequence for $I_{q}$, then there exist constants $K>0$ and $\delta>0$ such that

(a) $\left\|u_{n}\right\|_{X_{1}} \leq K, \quad \forall n \geq 1$,

(b) $\left|u_{n}\right|_{3} \geq \delta$ for all sufficiently large $n$, and

(c) there is a subsequence, still denoted by $u_{n}$, such that $\lim _{n \rightarrow \infty}\left\|u_{n}\right\|_{X_{1}}^{2}=\alpha>0$.

Proof. To prove statement (a), we observe that

$$
\begin{aligned}
& \frac{1}{2}\left(\beta \int\left(\partial_{x} u_{n}\right)^{2}+\gamma \int\left(D_{x}^{-1} u_{n}\right)^{2}-c \int u_{n}^{2}\right) \\
& =E\left(u_{n}\right)-c V\left(u_{n}\right)+\frac{1}{3} \int u_{n}^{3} \\
& \leq \sup _{n} E\left(u_{n}\right)-c q+C_{0}\left|u_{n}\right|_{2}^{\frac{5}{2}}\left\|u_{n}\right\|_{X_{1}}^{\frac{1}{2}} \\
& \leq C_{0}\left(1+\left\|u_{n}\right\|_{X_{1}}^{\frac{1}{2}}\right)
\end{aligned}
$$

where $C_{0}$ is a universal constant which is independent of $n$. Since $c<2 \sqrt{\gamma \beta}$, we have

$$
C_{0}\left(\left|\partial_{x} u_{n}\right|_{2}^{2}+\left|D_{x}^{-1} u_{n}\right|_{2}^{2}\right) \leq \beta \int\left(\partial_{x} u_{n}\right)^{2}+\gamma \int\left(D_{x}^{-1} u_{n}\right)^{2}-c \int u_{n}^{2}
$$

where the constant $C_{0}$ satisfies that $0<C_{0} \leq \min \left\{\frac{c \delta^{2}}{4}, \frac{\gamma^{2} \delta}{1+\gamma \delta}\right\}$ with $\delta=\frac{2 \beta}{c^{2}}-\frac{1}{2 \gamma}$. It then follows from the estimate (3.6) and (3.7) that $\left\|u_{n}\right\|_{X_{1}} \leq K$ where the constant $K=K(c, \beta, \gamma, q)$ but is independent of $n$.

To prove statement (b), we argue by contradiction. If no such constant $\delta$ exists, then

$$
\lim _{n \rightarrow \infty} \inf \left|u_{n}\right|_{3}^{3}=0 .
$$

So

$$
\begin{aligned}
I_{q} & =\lim _{n \rightarrow \infty}\left(\frac{\beta}{2} \int\left(\partial_{x} u_{n}\right)^{2}+\frac{\gamma}{2} \int\left(D_{x}^{-1} u_{n}\right)^{2}+\frac{1}{3} \int u_{n}^{3}\right) \\
& \geq-\frac{1}{3} \lim _{n \rightarrow \infty} \inf \left|u_{n}\right|_{3}^{3}=0,
\end{aligned}
$$

contradicting Lemma 3.2. 
To prove statement (c), using (a), we obtain that $\lim _{n \rightarrow \infty}\left\|u_{n}\right\|_{X_{1}}=\alpha$ exists for a subsequence $\left\{u_{n}\right\}$. It then follows from (b) that

$$
0<\delta^{3} \leq\left|u_{n}\right|_{3}^{3} \leq C_{0}\left|u_{n}\right|_{2}^{\frac{3}{2}}\left|D_{x}^{-1} u_{n}\right|_{2}^{\frac{1}{2}}\left|\partial_{x} u_{n}\right|_{2} \leq C_{0}\left\|u_{n}\right\|_{X_{1}}^{3} .
$$

It thus transpires that $\alpha>0$.

Lemma 3.4 (Subadditivity). For all $\mu_{1}, \mu_{2}>0$, suppose $\mu_{1}+\mu_{2}=\mu$. Then

$$
I_{\mu}<I_{\mu_{1}}+I_{\mu_{2}} \text {. }
$$

Proof. First we claim that for any $\mu_{0}, \mu>0$ with $\mu_{0}<\mu$,

$$
I_{\mu_{0}}>\left(\frac{\mu_{0}}{\mu}\right)^{\frac{5}{3}} I_{\mu} .
$$

To see this, associated to each function $u \in X_{1}$ with $V(u)=\mu$, the function $w(x)$ is defined by $w(x)=a u(b x)$ for $a, b>0$ to be chosen later. Then

$$
V(w)=\frac{a^{2} b^{-1}}{2} \int u^{2}=a^{2} b^{-1} \mu
$$

and

$$
E(w)=\frac{a^{2} b \beta}{2} \int u_{x}^{2}+\frac{a^{2} b^{-3} \gamma}{2} \int\left(D_{x}^{-1} u\right)^{2}+\frac{a^{3} b^{-1}}{3} \int u^{3} .
$$

Let $a^{2} b=a^{3} b^{-1}$, or, equivalently, $a=b^{2}$. Then

$$
E(w)=b^{5}\left(\frac{\beta}{2} \int u_{x}^{2}+\frac{b^{-4} \gamma}{2} \int\left(D_{x}^{-1} u\right)^{2}+\frac{1}{3} \int u^{3}\right)
$$

On the other hand, for any $\mu_{0}<\mu$, choose the constant $b$ by $b=\left(\frac{\mu_{0}}{\mu}\right)^{\frac{1}{3}}$. Then $b<1$ and $a^{2} b^{-1} \mu=\mu_{0}$, or $V(w)=\mu_{0}$ by (3.10). It then follows from $((3.12)$ that

$$
E(w)>b^{5} E(u)=\left(\frac{\mu_{0}}{\mu}\right)^{\frac{5}{3}} E(u) .
$$

This implies that

$$
I_{\mu_{0}} \geq\left(\frac{\mu_{0}}{\mu}\right)^{\frac{5}{3}} I_{\mu}
$$

As a consequence, for any $\mu_{1}, \mu_{2}>0$ satisfying $\mu_{1}+\mu_{2}=\mu$, we have

$$
I_{\mu_{1}}+I_{\mu_{2}} \geq\left[\left(\frac{\mu_{1}}{\mu}\right)^{\frac{5}{3}}+\left(\frac{\mu_{2}}{\mu}\right)^{\frac{5}{3}}\right] I_{\mu}>I_{\mu},
$$

which is the expected result.

Proof of Theorem 3.1. To show statement (1), we basically apply the concentration compactness lemma [Lio] with

$$
\rho_{n}=\left|D_{x}^{-1} u_{n}\right|^{2}+\left|u_{n}\right|^{2}+\left|\partial_{x} u_{n}\right|^{2}
$$

where $\left\{u_{n}\right\}$ is a minimizing sequence for $I_{q}$ with $\int \rho_{n}=\left\|u_{n}\right\|_{X_{1}}^{2} \longrightarrow \alpha>0$ as $n \rightarrow \infty$, as proved in Lemma 3.3(c). 
(i) The "vanishing" case is avoided. We argue by contradiction. Assume that "vanishing" occurs, that is, for any $r>0$,

$$
\lim _{n \rightarrow \infty} \sup _{x \in \mathbf{R}} \int_{x-r}^{x+r}\left(\left|D_{x}^{-1} u_{n}\right|^{2}+\left|u_{n}\right|^{2}+\left|\partial_{x} u_{n}\right|^{2}\right)=0 .
$$

Applying Sobolev's inequality, we obtain

$$
\begin{aligned}
\int_{x-r}^{x+r}\left|u_{n}\right|^{3} & \leq 4 \sqrt{2}\left(\int_{x-r}^{x+r}\left|u_{n}\right|^{2}\right)^{\frac{3}{4}}\left(\int_{x-r}^{x+r}\left|D_{x}^{-1} u_{n}\right|^{2}\right)^{\frac{1}{4}}\left(\int_{x-r}^{x+r}\left|\partial_{x} u_{n}\right|^{2}\right)^{\frac{1}{2}} \\
& \leq 4 \sqrt{2}\left(\sup _{x \in \mathbf{R}}\left(\int_{x-r}^{x+r}\left(\left|D_{x}^{-1} u_{n}\right|^{2}+\left|u_{n}\right|^{2}+\left|\partial_{x} u_{n}\right|^{2}\right)\right)\right)^{\frac{3}{2}} .
\end{aligned}
$$

Now, covering $\mathbf{R}$ by intervals with the length 1 , in such a way, each point in $\mathbf{R}$ is contained in at most two intervals. We get

$$
\int\left|u_{n}\right|^{3} \leq 8 \sqrt{2}\left(\sup _{x \in \mathbf{R}} \int_{x-r}^{x+r}\left(\left|D_{x}^{-1} u_{n}\right|^{2}+\left|u_{n}\right|^{2}+\left|\partial_{x} u_{n}\right|^{2}\right)\right)^{\frac{3}{2}} \longrightarrow 0
$$

as $n \rightarrow \infty$. So, it turns out that

$$
\begin{aligned}
I_{q} & =\lim _{n \rightarrow \infty}\left(\frac{\beta}{2} \int\left(\partial_{x} u_{n}\right)^{2}+\frac{\gamma}{2} \int\left(D_{x}^{-1} u_{n}\right)^{2}+\frac{1}{3} u_{n}^{3}\right) \\
& \geq \lim _{n \rightarrow \infty}\left(-\frac{1}{3} \int\left|u_{n}\right|^{3}\right)=0,
\end{aligned}
$$

which contradicts Lemma 3.2.

(ii) In the "dichotomy" case, we claim by following the idea from [Lio] that for some $0<\eta<\alpha$ and any $\epsilon>0$, there exists $\delta(\epsilon)>0$ (with $\delta(\epsilon) \rightarrow 0$ as $\epsilon \rightarrow 0$ ), two sequences $\left\{w_{n}\right\}$ and $\left\{v_{n}\right\}$ in $X_{1}$, and an integer $n_{0}>0$ such that for $n \geq n_{0}$,

$$
\begin{gathered}
\left\|w_{n}+v_{n}-u_{n}\right\|_{X_{1}} \leq \delta(\epsilon), \\
\left|\left\|w_{n}\right\|_{X_{1}}^{2}-\eta\right| \leq \delta(\epsilon), \\
\left|\left\|v_{n}\right\|_{X_{1}}^{2}-(\alpha-\eta)\right| \leq \delta(\epsilon), \\
\left.|| u_{n}\right|_{2} ^{2}-\left|w_{n}\right|_{2}^{2}-\left|v_{n}\right|_{2}^{2} \mid \leq \delta(\epsilon),
\end{gathered}
$$

and

$$
\operatorname{dist}\left(\operatorname{supp}\left(w_{n}\right), \operatorname{supp}\left(v_{n}\right)\right) \longrightarrow+\infty
$$

as $n \rightarrow \infty$. In fact, assume that "dichotomy" occurs, i.e. that

$$
\lim _{t \rightarrow+\infty} Q(t)=\eta<\alpha
$$

where for $t \geq 0$,

$$
Q(t)=\lim _{n \rightarrow+\infty} \sup _{x_{0} \in \mathbf{R}} \int_{x_{0}+B_{t}} \rho_{n}
$$

and $B_{R}$ denotes the ball of radius $R$ centered at 0 . Then for any fixed $\epsilon>0$, there are $R_{0}>0$ and $R_{n}>0$ with $R_{n} \nearrow+\infty$ and $x_{n} \in \mathbf{R}$ such that

$$
\eta \geq \int_{x_{n}+B_{R_{0}}}\left(\left|u_{n}\right|^{2}+\left|h_{n}\right|^{2}+\left|\partial_{x} u_{n}\right|^{2}\right) \geq \eta-\epsilon
$$


and $Q_{n}\left(2 R_{n}\right) \leq \eta+\epsilon$ for $n \geq n_{0}$, where $u_{n}=\partial_{x} h_{n}$ and

$$
Q_{n}(t)=\sup _{x_{0} \in \mathbf{R}} \int_{x_{0}+B_{t}}\left(\left|u_{n}\right|^{2}+\left|h_{n}\right|^{2}+\left|\partial_{x} u_{n}\right|^{2}\right) .
$$

It then follows that

$$
\int_{R_{0} \leq\left|x-x_{0}\right| \leq 2 R_{n}}\left(\left|u_{n}\right|^{2}+\left|h_{n}\right|^{2}+\left|\partial_{x} u_{n}\right|^{2}\right) \leq 2 \epsilon .
$$

Let $\xi$ and $\theta \in C_{0}^{\infty}(\mathbf{R})$ such that $0 \leq \xi \leq 1,0 \leq \theta \leq 1, \xi \equiv 1$ on $B_{1}$, supp $\xi \subset B_{2}, \theta \equiv 1$ on $\mathbf{R} \backslash B_{2}$, and supp $\theta \subset \mathbf{R} \backslash B_{1}$. Define $\xi_{n}=\xi\left(\frac{. x_{n}}{R_{1}}\right)$ and $\theta_{n}=\theta\left(\frac{\cdot-x_{n}}{R_{n}}\right)$. Now let us consider

$$
w_{n}=\partial_{x}\left(\xi_{0}\left(h_{n}-a_{n}\right)\right), \quad v_{n}=\partial_{x}\left(\theta_{n}\left(h_{n}-b_{n}\right)\right)
$$

where $a_{n}$ and $b_{n}$ are sequences which will be chosen later. Moreover, let us set

$$
w_{n}^{1}=D_{x}^{-1} w_{n}=\xi_{n}\left(h_{n}-a_{n}\right)
$$

and

$$
v_{n}^{1}=D_{x}^{-1} v_{n}=\theta_{n}\left(h_{n}-b_{n}\right)
$$

Then we deduce that

$$
\left|w_{n}^{1}+v_{n}^{1}-h_{n}\right|_{2} \leq\left|\xi_{n}\left(h_{n}-a_{n}\right)\right|_{2}+\left|\theta_{n}\left(h_{n}-b_{n}\right)\right|_{2}+\sqrt{2 \epsilon}
$$

and

$$
\begin{aligned}
\left|\xi_{n}\left(h_{n}-a_{n}\right)\right|_{2} & =\left(\int_{R_{1} \leq\left|x-x_{n}\right| \leq 2 R_{1}}\left|\xi_{n}\right|^{2}\left|h_{n}-a_{n}\right|^{2}\right)^{1 / 2} \\
& \leq\left|\xi_{n}\right|_{\infty}\left(\int_{R_{1} \leq\left|x-x_{n}\right| \leq 2 R_{1}}\left|h_{n}-a_{n}\right|^{2}\right)^{1 / 2} .
\end{aligned}
$$

Now choosing

$$
a_{n}=\frac{1}{\operatorname{Vol}\left(\Omega_{x_{0}, R_{1}}\right)} \int_{R_{1} \leq\left|x-x_{0}\right| \leq 2 R_{1}} h_{n}=m_{R_{1}}\left(h_{n}\right)
$$

with $\Omega_{x_{0}, R_{1}}=\left\{x \in \mathbf{R} ; R_{1}<\left|x-x_{0}\right|<2 R_{1}\right\}$, we have

$$
\left|\xi_{n}\left(h_{n}-a_{n}\right)\right|_{2} \leq C\left(\int_{R_{1} \leq\left|x-x_{0}\right| \leq 2 R_{1}}\left|u_{n}\right|^{2}\right)^{1 / 2} \leq C \sqrt{\epsilon}
$$

In the same way, choosing $b_{n}=m_{R_{n}}\left(h_{n}\right)$ leads to the bound

$$
\left|\theta_{n}\left(h_{n}-b_{n}\right)\right|_{2} \leq C\left(\int_{R_{n} \leq\left|x-x_{0}\right| \leq 2 R_{n}}\left|u_{n}\right|^{2}\right)^{1 / 2} \leq C \sqrt{\epsilon} .
$$

Hence the desired estimate on $\left|w_{n}^{1}+v_{n}^{1}-h_{n}\right|_{2}$ can be obtained by the above inequalities and the estimate on $\left|w_{n}+v_{n}-u_{n}\right|_{2}$ is obtained in the same way. Attention is now turned to estimate $\left|\partial_{x} w_{n}+\partial_{x} v_{n}-\partial_{x} u_{n}\right|_{2}$. It is found that

$$
\begin{aligned}
\mid \partial_{x} w_{n}+ & \partial_{x} v_{n}-\left.\partial_{x} u_{n}\right|_{2}=\left|\partial_{x}^{2}\left(\xi\left(h_{n}-a_{n}\right)\right)+\partial_{x}^{2}\left(\theta_{n}\left(h_{n}-b_{n}\right)\right)-\partial_{x}^{2} h_{n}\right|_{2} \\
& \leq\left|\left(\partial_{x}^{2} \xi_{n}\right)\left(h_{n}-a_{n}\right)\right|_{2}+\left|\left(\partial_{x}^{2} \theta_{n}\right)\left(h_{n}-b_{n}\right)\right|_{2}+\left|\left(1-\xi_{n}-\theta_{n}\right) \partial_{x} u_{n}\right|_{2} \\
& +2\left|\left(\partial_{x} \xi_{n}\right) u_{n}\right|_{2}+2\left|\left(\partial_{x} \theta_{n}\right) u_{n}\right|_{2} .
\end{aligned}
$$


The first three terms in the right hand side of the above inequality are bounded as the preceding ones. For the last two terms, we have

$$
\left|\left(\partial_{x} \xi_{n}\right) u_{n}\right|_{2} \leq\left|\partial_{x} \xi_{n}\right|_{\infty}\left(\int_{R 1 \leq\left|x-x_{0}\right| \leq 2 R_{1}}\left|u_{n}\right|^{2}\right)^{1 / 2} \leq C \sqrt{\epsilon}
$$

All the other terms in (ii) can be estimated in a similar way and the last bound follows from the first one, the fact that supp $w_{n}^{1} \cap \operatorname{supp} v_{n}^{1}=\emptyset$ and the injection of $X_{1}$ into $L^{2}$.

Now taking subsequences if necessary, we may assume

$$
\lim _{n \rightarrow \infty} \int_{\mathbf{R}}\left(w_{n}\right)^{2}=\lambda_{1}(\epsilon), \quad \lim _{n \rightarrow \infty} \int_{\mathbf{R}}\left(v_{n}\right)^{2}=\lambda_{2}(\epsilon)
$$

with $\left|\lambda_{1}(\epsilon)+\lambda_{2}(\epsilon)-q\right| \leq \delta(\epsilon)$. Then in view of the estimates in (ii), we deduce that $\lim _{\epsilon \rightarrow 0} \lambda_{1}(\epsilon)>0, \lim _{\epsilon \rightarrow 0} \lambda_{2}(\epsilon)>0$ and

$$
I_{\lambda_{1}}+I_{\lambda_{2}} \leq I_{q}+\delta(\epsilon)
$$

We then reach a contradiction by letting $\epsilon$ tend to zero and the subadditivity property of $I_{q}$ in Lemma 3.4. This rules out the "dichotomy" case.

(iii) The only remaining possibility is then the "concentration" of the sequence $\left\{u_{n}\right\}$ up to translations. That is, there exists a sequence $\left\{y_{n}\right\}$ with $y_{n} \in \mathbf{R}$ for all $n \geq 1$, such that for any $\epsilon>0$, there exists $r>0$ and $n_{0}>0$, for all $n \geq n_{0}$, i.e.

$$
\int_{y_{n}-r}^{y_{n}+r}\left(\left|D_{x}^{-1} u_{n}\right|^{2}+\left|u_{n}\right|^{2}+\left|\partial_{x} u_{n}\right|^{2}\right) d x \geq \alpha-\epsilon .
$$

This implies that for $n$ large enough,

$$
\int_{y_{n}-r}^{y_{n}+r}\left|u_{n}\right|^{2} \geq \int_{\mathbf{R}}\left|u_{n}\right|^{2}-2 \epsilon .
$$

Since $u_{n}$ is bounded in $X_{1}$, one may assume that a subsequence of $u_{n}$ (still denoted by $u_{n}$ ) converges weakly in $X_{1}$ to some $g \in X_{1}$. It then follows that

$$
\int_{\mathbf{R}}|g|^{2} \leq \lim _{n \rightarrow \infty} \inf \int_{\mathbf{R}}\left|u_{n}\right|^{2} \leq \lim _{n \rightarrow \infty} \inf \int_{y_{n}-r}^{y_{n}+r}\left|u_{n}\right|^{2}+2 \epsilon .
$$

On the other hand, using the relative compactness of the injection $X_{1} \subset L_{\text {loc }}^{2}$, it then follows from (3.21) that some subsequence of $\left\{u_{n}\left(\cdot+y_{n}\right)\right\}$ with $y_{n} \in \mathbf{R}$ converges strongly in $L_{2}(\mathbf{R})$. By interpolation

$$
|u|_{3}^{3} \leq 4 \sqrt{2}|u|_{2}^{\frac{3}{2}}\|u\|_{X_{1}}^{\frac{3}{2}}
$$

and one obtains that the sequence $u_{n}\left(\cdot+y_{n}\right)$ also converges to $g$ strongly in $L_{3}$. As a consequence, it follows that

$$
E(g) \leq \lim _{n \rightarrow \infty} \inf E\left(u_{n}\right) \leq E(g)=I_{q} .
$$

This shows that $g$ is a solution of $I_{q}$ and the set $\Sigma_{q}$ is not empty. Note that from the above proof, we also obtain that a subsequence of $\left\{u_{n}\left(\cdot+y_{n}\right)\right\}$ is strongly convergent to $g \in X_{1}$. This proves statement (2). Now suppose that statement (3) does not hold. Then there exists a subsequence $\left\{u_{n_{k}}\right\}$ of $\left\{u_{n}\right\}$ and a small number $\delta>0$ such that

$$
\inf _{g \in \Sigma_{g}, y \in \mathbf{R}}\left\|u_{n_{k}}(\cdot+y)-g\right\|_{X_{1}} \geq \delta
$$


for all $k \geq 1$. Since $\left\{u_{n_{k}}\right\}$ is itself a minimizing sequence for $I_{q}$ from statement (1), it follows that there exists a sequence $\left\{y_{k}\right\}$ and $g_{0} \in \Sigma_{q}$ such that

$$
\lim _{k \rightarrow \infty}\left\|u_{n_{k}}\left(\cdot+y_{k}\right)-g_{0}\right\|_{X_{1}}=0 .
$$

This contradicts (3.22). For statement (4), we note that the functionals $E$ and $V$ are invariant under translations. This implies that $g(\cdot-y) \in \Sigma_{q}$ for any $y \in \mathbf{R}$ provided $g \in \Sigma_{q}$. It then turns out that

$\lim _{n \rightarrow \infty} \inf _{g \in \Sigma_{q}}\left\|u_{n}-g\right\|_{X_{1}} \leq \lim _{n \rightarrow \infty} \inf _{g \in \Sigma_{q}}\left\|u_{n}-g(\cdot-y)\right\|_{X_{1}}=\lim _{n \rightarrow \infty} \inf _{g \in \Sigma_{q}}\left\|u_{n}(\cdot+y)-g\right\|_{X_{1}}=0$.

This completes the proof of statement (4). For statement (5), it follows from Proposition 2.3 that $\varphi_{c} \in G_{c}$ and therefore if $g \in \Sigma_{q}$, then

$$
L_{c}(g) \leq L_{c}\left(\varphi_{c}\right) \leq L_{c}(f)
$$

for any $f \in \Omega_{c}$. By the definition of $\Sigma_{q}$ and the Lagrange multiplier principle, for each $g \in \Sigma_{q}$ there exists $\lambda \in \mathbf{R}$ such that

$$
\delta E(g)=\lambda \delta V(g)
$$

where the Fréchet derivatives $\delta E(g)$ and $\delta V(g)$ are given by

$$
\delta E(g)=-\beta g_{x x}-\gamma D_{x}^{-2} g+g^{2}
$$

and

$$
\delta V(g)=g
$$

Therefore $g$ solves (1.8) with the wavespeed $\lambda$. In view of (3.23), we have

$$
\int \beta g_{x}^{2}+\gamma\left(D_{x}^{-1} g\right)^{2}+g^{3}=\lambda \int g^{2}
$$

As a consequence, we obtain from (3.24) that

$$
P(g)+c \int g^{2}=\lambda \int g^{2} .
$$

In view of $P(g)=0$, it is concluded that $\lambda=c$ as claimed and the proof of Theorem 3.1 is complete.

4. Dynamical stability. We are now in the position to prove the dynamical stability result, Theorem 1.3. It is an immediate consequence of Theorem 3.1.

Proof of Theorem 1.3. Suppose the set $\Sigma_{q}$ is not stable. Then there exists a real number $\epsilon>0$, a sequence $\left\{\phi_{n}\right\}$ in $X_{1}$, and $t_{n} \geq 0$ such that

$$
\inf _{g \in \Sigma_{q}}\left\|\phi_{n}-g\right\|_{X_{1}}<\frac{1}{n}
$$

and

$$
\inf _{g \in \Sigma_{q}}\left\|u_{n}\left(\cdot, t_{n}\right)-g\right\|_{X_{1}} \geq \epsilon
$$


for all $n \geq 1$, where $u_{n}(x, t)$ solves (1.1) with $u_{n}(x, 0)=\phi_{n}$. Since $\phi_{n} \rightarrow g$ in $X_{1}$ and since $E(g)=I_{q}$ and $V(g)=q$ for some $g \in \Sigma_{q}$, we have $E\left(\phi_{n}\right) \rightarrow I_{q}$ and $V\left(\phi_{n}\right) \rightarrow q$, as $n \rightarrow \infty$. But $E\left(u_{n}\right)=E\left(\phi_{n}\right) \rightarrow I_{q}$ and $V\left(u_{n}\right)=V\left(\phi_{n}\right) \rightarrow q$. Choose

$$
\alpha_{n}=\left(\frac{q}{V\left(\phi_{n}\right)}\right)^{\frac{1}{2}} \text {. }
$$

Then $\alpha_{n} \rightarrow 1$ and $V\left(\alpha_{n} u_{n}\right)=q$ for all $n$, and $u_{n}\left(\cdot, t_{n}\right)$ is uniformly bounded, say, by $M$. Therefore, $f_{n}=\alpha_{n} u_{n}$ is a minimizing sequence of $I_{q}$. It then follows from Theorem 3.1 that for all $n$ sufficiently large there exists $g_{n} \in \Sigma_{q}$ such that $\left\|f_{n}-g_{n}\right\|_{X_{1}}<\frac{\epsilon}{2}$. But then

$$
\begin{aligned}
\epsilon & \leq\left\|u\left(\cdot, t_{n}\right)-g_{n}\right\|_{X_{1}} \leq\left\|u_{n}\left(\cdot, t_{n}\right)-f_{n}\right\|_{X_{1}}+\left\|f_{n}-g_{n}\right\|_{X_{1}} \\
& \leq\left|1-\alpha_{n}\right|\left\|u_{n}\left(\cdot, t_{n}\right)\right\|_{X_{1}}+\frac{\epsilon}{2}<\left|1-\alpha_{n}\right| M+\frac{\epsilon}{2}
\end{aligned}
$$

and taking $n \rightarrow \infty$ gives $\epsilon \leq \frac{\epsilon}{2}$, a contradiction. This completes the proof of Theorem 1.3 .

Acknowledgments. The author would like to thank Y. Guo for stimulating discussions. The author also thanks the referee for valuable comments and suggestions.

\section{REFERENCES}

[Be] Benilov, E. S., On the surface waves in a shallow channel with an uneven bottom, Stud. Appl. Math., 87(1992), 1-14. MR1165932 (93b:76009)

[BrLi] Brézis, H. and Lieb, E., A relation between pointwise convergence of functions and convergence of functionals, Proc. Amer. Math. Soc., 88 (1993), 437-477. MR699419 (84e:28003)

[BoSa] de Bouard, A. and Saut, J. C., Solitary waves of generalized Kadomtsev-Petviashvili equations, Ann. Inst. H. Poincaré Anal. Non Linéaire, A14(1997), 211-236. MR1441393 (98a:35115)

[CaLi] Cazenave, T. and Lions, P. L., Orbital stability of standing waves for some nonlinear Schrödinger equations, Comm. Math. Phys., 85 (1982), 549-561. MR677997 (84i:81015)

[FrLiLo] Fröhlich, J., Lieb, E. H., and Loss, M., Stability of coulomb systems with magnetic fields I. The one electron atom, Commun. Math. Phys., 104(1986), 251-270. MR836003 (88a:81023a)

[GaSt] Galkin, V. N. and Stepanyants, Y. A., On the existence of stationary solitary waves in a rotating field, J. Appl. Math. Mech., 55 (1991), 939-943. MR1150448 (92j:76096)

[GiGrSt] Gilman, O. A., Grimshaw, R., and Stepanyants, Y. A., Approximate and numerical solutions of the stationary Ostrovsky equation, Stud. Appl. Math., 95(1995), 115-126. MR1342073 (96c:76018)

[Gr] Grimshaw, R., Evolution equations for weakly nonlinear long internal waves in a rotating fluid, Stud. Appl. Math., 73(1985), 1-33. MR797556 (87a:76029)

[KaPe] Kadomtsev, B. B. and Petviashvili, V. I., On the stability of solitary waves in weakly dispersive media, Sov. Phys. Dokl., 15(6)(1970), 539-541.

[Lie] Lieb, E. H., On the lowest eigenvalue of Laplacian for the intersection of two domains, Invent. Math., 74(1983), 441-448. MR724014 (85e:35090)

[Lio] Lions, P. L., The concentration compactness principle in the calculus of variations. The locally compact case, Part 1 and Part 2, Ann. Inst. H. Poincaré, Anal. Nonlinéaire, 1(1984), 109-145, 223-283. MR778970 (87e:49035a); MR0778974 (87e:49035b)

[LiVa] Liu, Yue and Varlamov, V., Stability of solitary waves and weak rotation limit for the Ostrovsky equation, J. Differential Equations, 203(2004), 159-183. MR2070389 (2005f:76022)

[MiGaKr] Miura, R. M., Gardner, C. S., and Kruskal, M. D., Korteweg-de Vries equation and generalizations II, Existence conservation laws and constant of motion, J. Math. Phys., 9(1968), 1204-1209. MR0252826 (40:6042b)

[Os] Ostrovsky, L. A., Nonlinear internal waves in a rotating ocean, Oceanologia, 18(1978), 181191. 
[OsSt] Ostrovsky, L. A. and Stepanyants, Y. A., Nonlinear surface and internal waves in rotating fluids, Research Reports in Physics, Nonlinear Waves 3, Springer, Berlin, Heidelberg, 1990.

[VaLi] Varlamov, V. and Liu, Yue, Cauchy problem for the Ostrovsky equation, Discrete Dynam. Systems, 10(2004), 731-751. MR2018877 (2004j:35235) 